\title{
THE W (Sn-Mo)-SPECIALIZED CATINGA SUITE AND OTHER GRANITOIDS OF THE BRUSQUE GROUP, NEOPROTEROZOIC OF THE STATE OF SANTA CATARINA, SOUTHERN BRAZIL
}

\author{
NEIVALDO ARAÚJO DE CASTRO*, MIGUEL ÂNGELO STIPP BASEI** AND \\ ÁLVARO PENTEADO CRÓSTA***
}

\begin{abstract}
RESUMO A SUÍTE ESPECIALIZADA EM W (Sn-Mo) DE CATINGA E OUTROS GRANITÓIDES DO GRUPO BRUSQUE, NEOPROTEROZOICO DO ESTADO DE SANTA CATARINA BRASIL MERIDIONAL Dados petrográficos, geoquímicos e isotópicos de granitóides tardi a pós-tectônicos, intrusivos no Grupo Brusque, Santa Catarina, são apresentados neste artigo. Os granitóides intrusivos no domínio sul do Grupo Brusque (GIDS) são geralmente acinzentados, biotita, quando presente é praticamente o único máfico, apresentam características redutoras. Os granitóides atingem as composições mais ácidas, são levemente alcalinos, transicionais entre meta e peraluminosos Associado aos GIDS parece ocorrer um corpo sub-vulcânico ácido, conhecido como sub-vulcânicas Ribeirão da Velha, o qual, por suas características petrográficas e geoquímicas possui boa chance de estar associado geneticamente aos GIDS. Na suíte Catinga piracipnarnento dos LREE em relação aos HREE é incipiente, e geralmente associa-se a uma anomalia negativa de Eu pronunciada. As razões ${ }^{87} \mathrm{Sr} /{ }^{86} \mathrm{Sr}$ reportadas na literatura para os GIDS são em torno de 0,721 (granitóide São João Batista) e a única idade modelo IDM disponível (granitóide Valsungana) aponta para a presença de granitóides oriundos de uma crosta continental com idade em torno de 2020 Ma. Rochas gnáissico-migmatíticas como as que ocorrem na porção leste do domínio do Grupo Brusque e como enclaves no granitóide Nova Itália parecem ser fortes candidatas como possíveis protólitos para os GIDS As poucas informações disponíveis para os granitóides intrusivos no domínio norte do Grupo Brusque (GIDN) evidenciam a presença de hornblenda granitóides com feldspatos potássicos róseos (pluton Faxinai) a levemente róseos com biotita (pluton Guabiruba), mais enriquecidos em $\mathrm{K}_{2} \mathrm{O}$ e formadas em condições oxidantes que os GIDS, sendo o fracionamento dos LREE em relação aos HREE bastante pronunciado e associado a anomalia negativa de Eu incipiente. Aos GIND aventa-se a hipótese de estarem associadas algumas concentrações de $\mathrm{Au}$ situadas neste domínio, uma vez que as características apresentadas por estes granitóides parecem propiciar mais a concentração deste metal que aos associados aos GIDS. Diferenças nos protólitos e processos evolutivos de sul para norte atuantes em condiç̃es tardi a pós-colisionais sob a influência de uma crosta continental espessa, durante o Neoproterozóico, são apontados como prováveis responsáveis
\end{abstract} pela geração da variedade de granitóides observados no domínio do Grupo Brusque.

Palavras-chave:Brasil, Neoproterozóico, minerais metálicos, petrografia, geoquímica.

ABSTRACT The petrographic, geochemical and isotopic data on the granitoids intrusive in the Brusque Group, State of Santa Catarina, southern Brazil, are presented in this paper. These are late- to post-tectonic rocks, being the most evolved ones those that constitute the Catinga Suite. The granitoids intrusive in the southern domain of the Brusque Group are grayish rocks, in which biotite (when present) is practically the only mafic mineral. They are rather reduced, slightly alkalic, transitional between meta-and peraluminous. An acid subvolcanic pluton, the Ribeirão da Velha pluton, has similar petrographic and geochemical characteristics, and seems to be genetically related to these granitoids. LREE fractionation relative to $\mathrm{HREE}$ is incipient for the Catinga suite, and the REE patterns showing strong negative Eu anomaly. Initial ${ }^{87} \mathrm{Sr} /{ }_{86} \mathrm{Sr}$ ratios, from the literature for these granitoids are around 0.721 (São João Batista granitoid) and the only available IDM model age (Valsungana granitoid) points to a genesis from a continental crust of $c a .2020$ Ma old. Gneiss-migmatitic rocks such as those that occur in the eastern portion of Brusque Group domain and as enclaves in the Nova Itália granitoid are strong candidates for protoliths of these granitoids. Little available information on the granitoids intrusive in the northern domain of the Brusque Group reports the presence of pink K-feldspar hornblende granitoids (Faxinai pluton) and light pink K-feldspar biotite granitoid (Guabiruba pluton). These granitoids are more enriched in $\mathrm{K}$ and more oxidized than those in the southern domain, and the LREE fractionation relative to HREE is strong, with the REE patterns showing incipient negative Eu anomaly. It is suggested that part of the Au concentrations found in the northern domain may be genetically associated with the granitoids, since they present characteristics favorable to promote $\mathrm{Au}$ mineralizations than the granitoids in the southern domain. Distinct protoliths and evolutionary processes from south to north within the Brusque Group domain, late to post-collisional conditions and the influence of a thick continental crust during the Neoproterozoic are the factors responsible for the generation of such a variety of granitoids in Brusque Group domain.

Keywords: Brazil, Neoproterozoic, metalllic menerals, petrography, geochemistry.

INTRODUCTION A review of the literature, as well as new geochemical data from the Catinga Suite granitoids (CS) and other granitoids intrusive in the metamorphosed volcano-sedimentary sequence of the Brusque Group domain (BCD; Fig. 1), State of Santa Catarina, southern Brazil, is presented in this work. Features showed include petrographic and geochemical data, and a discussion on the petrogenesis and metallogenetic aspects of the granitoids.

PREVIOUS WORKS The first works on the intrusive granitoids of the Brusque Group domain date back to the 60's, when Schulz Jr. \& Albuquerque (1969) introduced the designations Valsungana granodiorite and Guabiruba granite for granitoids intrusive in the southern Brusque Group. Later, Trainini et al. (1978) used the terminology Valsungana and Guabiruba suites. The Valsungana suite comprises the major batholiths known in Brusque Group domain, which are composed of calc-alkalic granitoids with microcline megacrysts in a coarse-grained matrix, predominantly of granodioritic and, less common, granitic and quartz-monzonitic compositions. Smaller plutons are included in the Guabiruba suite, which are composed of fine-grained equigranular quartz-monzonites and granites with alkalic affinity. Trainini et al. (op cit.) proposed a crustal origin and metallogenetic specialization ( $\mathrm{W} \pm \mathrm{Sn}-\mathrm{Mo}$ ) for the Catinga granitoid, a pluton intrusive in the southern domain of the Brusque Group, part of the Guabiruba Suite

Basei (1985) obtained a Neoproterozoic age for some granitoids intrusive in the Brusque Group. The oldest ages were found for the Valsungana suite plutons, which range from $647 \pm 12 \mathrm{Ma}$ (U-Pb in zircons) to $500 \mathrm{Ma}(\mathrm{K}-\mathrm{Ar})$. Rb-Sr ages are in the same range, and initial ${ }^{87} \mathrm{Sr} /{ }^{86} \mathrm{Sr}$ ratios are high, ranging from 0.7105 to 0.721 (the latter found for the São João Batista granitoid). Based on available petrography, chemical and isotopic informations, Basei (1985) concluded that the great majority of the granitoids in the Brusque Group have S-type affinity, using the Chappel \& White (1974) classification. According to this author, presence of large volume of S-type granitoids rule out the involvement of oceanic crust in the genesis of the Brusque Group during the Neoproterozoic convergence in the region.

Mantovani et al. (1987) obtained a $t_{\mathrm{DM}} \mathrm{Nd}$ model age of 2,020 Ma and a low $\varepsilon_{\mathrm{Nd}}(-14.74)$ for a sample from the Valsungana suite, attesting an old continental crust source for the granitoids.

Caldasso et al. (1988) introduced the term Faxinai granitoid to designate a biotite-hornblende syenite pluton that occurs south of the shear zone of the Ribeirão da Prata Belt (ZCFRP, Fig. 1). These authors pointed out the presence of more evolved granitoid compositions within the Brusque Group, such as biotite monzo- to syenogranites in the Valsungana Suite and predominating syenogranitic terms in the Guabiruba Suite. These authors also proposed the term Indaiá Facies to describe the granitoid with transitional characteristics between Valsungana and Guabiruba (a few K-feldspar phenocrysts).

Petrographic and geochemical studies performed by Castro \& Basei (1995), allowed a better individualization of the suites and plutons in the Brusque Group, and Castro (1997) did a detailed petrological and geochemical work on these plutons, which is summarized in this paper.

METALLIC MINERAL RESOURCES IN THE BRUSQUE GROUP The metallic mineral resources present in Brusque Group are restricted to $\mathrm{Au}$ and $\mathrm{W}$. Au occurs in the Gaspar, Botuverá and Canelinha regions and economic $\mathrm{W}$ concentrations are presently found only in the old Cerro da Catinga mine region. The relationships

* State University of Campinas, Institute of Geosciences. C.P.6152; 13083-970, Campinas, SP, Brazil (neivaldo@ufc.br))

** University of São Paulo, Institute of Geosciences. C.P. 11348; 05422-970, São Paulo, SP, Brazil (baseimas@usp.br)

*** State University of Campinas, Institute of Geosciences. C.P. 6152; 13083-970, Campinas, SP, Brazil (alvaro@ige.unicamp.br) 


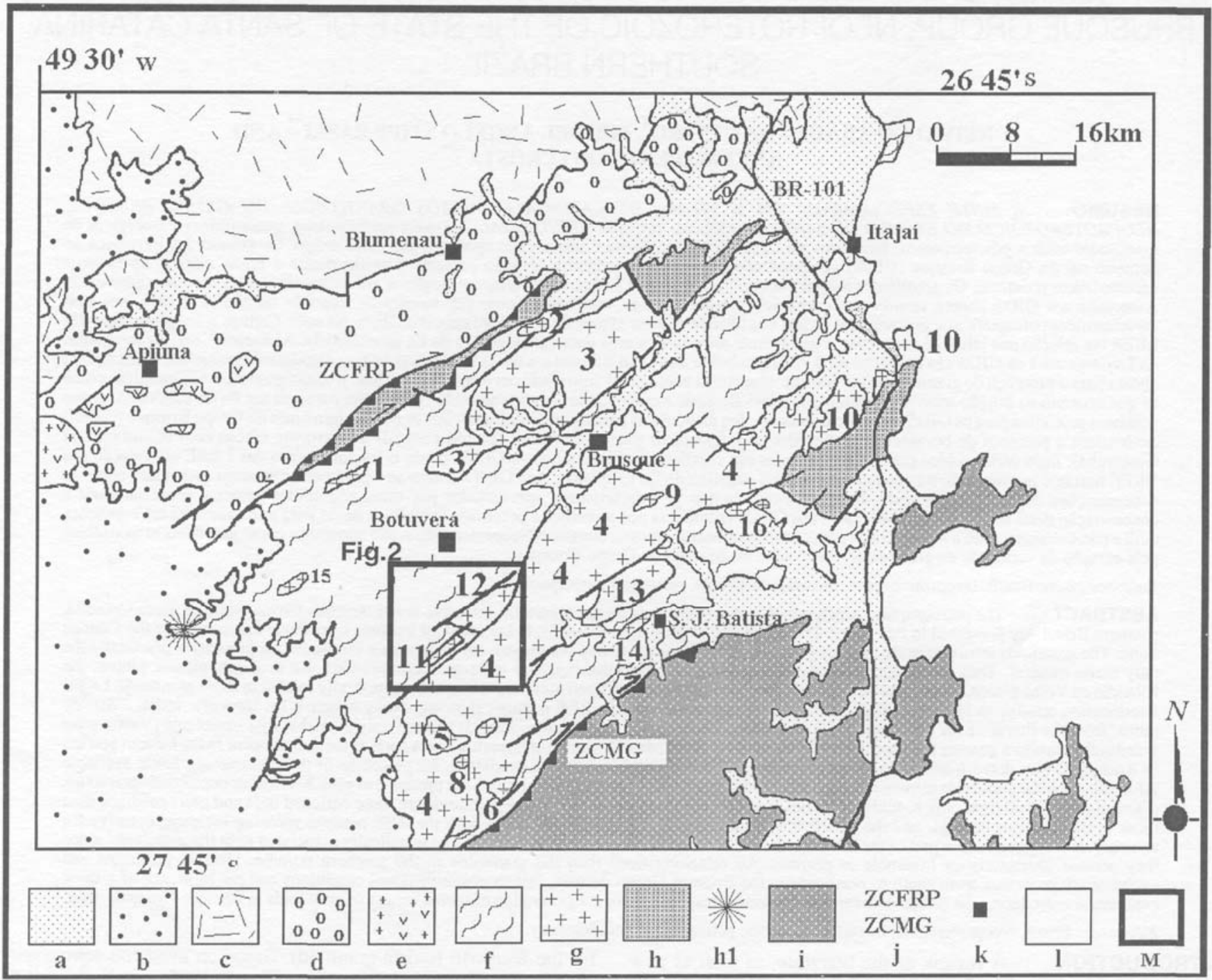

Figure 1 - General geology of the Brusque Group, Santa Catarina State, southern Brazil, modified from Basel (1985) and Caldasso et al. (1994a and b). a: Quaternary deposits; b: Phanerozoic Sedimentary basin (Paraná Basin); c: Luis Alves Craton (granulites and granulitic-gneisses); DOM FELICIANO BELT - EXTERNAL DOMAIN: d: Itajai Molasse Sequence; e: Subida Suite (acid plutonism and volcanism); DOM FELICIANO BELT - INTERMEDIARY DOMAIN: $f$ : Brusque Group (schists, marbles, phyllites, quartzites with basic and acid metavolcanic lenses); g: intrusive granitoids (l-Faxinai, 2- Gaspar, 3-Giiabiruba, 4-Valsungana, 5-Morro Pelado, 6-Rio do Alho, 7-Beija-Flor, 8-Santo Aleixo, 9-Nova Itália, 10-Morro do Boi, 11-Catinga, 12-Tijucas, 13-NovaTrento, 14-São João Batista, 15-Barrado Areia) and 16-Campo Novo Segundo; $\boldsymbol{h}$ : Quartz-feldspatic gneisses and migmatites, h1: Presidente Nereu occurrence; DOM FELICIANO BELT - INTERNAL DOMAIN: i: granitoids, subordinate gneisses and migmatites; $\mathbf{j}$ : Major Shear Belts: ZCRP (Ribeirão da Prata) and ZCMG (Major Gercino); $\boldsymbol{k}$ : major cities; l: Atlantic Ocean; BR-101: highway.

between $\mathrm{Au}$ concentrations and the granitoids in the northern domain of the Brusque Group are still not clear. However, as it will be seen later, great part of the Au concentrations in the northern domain of the Brusque Group may be genetically related to granitoids, generated by fluids originated from the intrusions. Castro (1997) concluded that the granitoids have played the role of important heat sources to allow remobilization of $\mathrm{Au}$ contained in the adjacent volcanosedimentary sequences.

Wolframite-bearing quartz veins are found in two places in the Brusque Group, one of them immediately north of Botuverá (Ribeirão do Russo field of occurrences) and another one in the vicinities of the Catinga Suite (Fig 2). However, economically exploited W is found only in the old Cerro da Catinga mine, in which $\mathrm{W}$ was economically exploited until the end of the 40's. The N60-70E direction structurally controls the wolframite-bearing quartz veins (Trainini et al. 1978, Silva \& Krebs 1980, Silva et al. 1986). According to these authors the measured ore reserve sum $6200 \mathrm{t}$ for $c a .135 \mathrm{t}$ of contained $\mathrm{WO}_{3}$. A series of Sn and Mo occurrences (Fig. 2) are also found in this region. The Catinga suite specialization of metals of crustal affinity such as $\mathrm{W}$ and $\mathrm{Sn}$ is always cited in the literature concerned to the metallogenetic potential associated with the granitoids of the Brusque Group (e.g. Issler 1987).

PETROGRAPHY Table 1 summarizes the petrographic features of 94 samples of the Catinga Suite and other granitoids intrusive in the Brusque Group.

Macroscopically, the granitoids are gray to whitish gray, hipidiomorphic equigranular, and rarely inequigranular, sometimes presenting pink K-feldspar. The Catinga, São João Batista and Campo Novo granitoids are medium- to fine-grained rocks, while other plutons are medium- to coarse grained. Late, brittle fractures are observed in most plutons.

Modal proportions of quartz, alkali feldspar and plagioclase granitoids in the Brusque Group are represented in the QAP diagram of Fig. 
3. Samples from the Catinga pluton plot close to apex A (alkali feldspar), predominantly in the field of syenogranite, alkali-feldspar granite, and less often, of quartz syenite. Rocks from the Catinga suite plot on the most differentiated end of a compositional trend that start with Valsungana quartz monzodiorites and quartz monzogabbros, in the Fig. 3 lying in the field of high-K calc-alkalic series, according to Lameyre \& Bowden (1982). The Faxinai hornblende quartz syenite, which occurs in the northern domain of the Brusque Group, plot outside the field for other plutons in the Brusque Group, suggesting they are little differentiated rocks of alkalic series of aluminous provinces

Brown to reddish-brown biotite is present in all plutons in low proportions (up to $3 \%$ ), showing alteration to chlorite and fine-grained opaque minerals. Primary as well as secondary (from alteration of feldspar) muscovite is found in modal amounts up to $5 \%$, in all plutons, except for the Faxinai suite and the Campo Novo Segundo granitoid, in the northern domain of the Brusque Group, which, instead, show hornblende. The main accessory minerals are zircon, apatite, titanite, tourmaline and opaque minerals; allanite and garnet (?) are less common.

Late minerals present are fluorite (usually in the microfractures of feldspars and quartz), sericite, epidote, fine-grained opaque and clay minerals. Carbonates are rare, and wolframite (?) is observed only in the Catinga granitoids.

GEOCHEMISTRY Table 2 displays major and trace element analyses for some granitoids in the Brusque Group, carried out in several laboratories. Harker-type diagrams (Fig 4) show that the Catinga granitoids, which are silica rich, plot in the extension of the trends formed by other granitoids in the Brusque Group. They show lower $\mathrm{CaO}$ and $\mathrm{TiO}_{2}$ and higher $\mathrm{Na}_{2} \mathrm{O}$ and $\mathrm{Y}$ contents than the other granitoids in the region. The geochemical behavior of the Ribeirão da Velha subvolcanic rocks is similar to that of the Catinga granitoid, except for their higher $\mathrm{K}_{2} \mathrm{O}, \mathrm{MgO}, \mathrm{Zr}, \mathrm{Rb}$, Th and $\mathrm{U}$ contents and slight $\mathrm{Na}_{2} \mathrm{O}$ and $\mathrm{CaO}$ depletion (Table 2).

The plutons present two main REE patterns (Figs. 5 and 6). The first pattern (Fig. 5) comprises the Faxinai, Valsungana, Morro Pelado suites and Ribeirão da Velha subvolcanic rocks. It is characterized by a pronounced fractionation between LREE and HREE $\left(\mathrm{La}_{\mathrm{n}} / \mathrm{Yb}_{\mathrm{n}}=50\right.$ for the Faxinai pluton and $\mathrm{La}_{\mathrm{n}} / \mathrm{Yb}_{\mathrm{n}} \approx 15$ for the Valsungana suite) and by slightly negative Eu anomaly. The second pattern (Fig. 6) is characteristic for the Catinga suite (Fig. 6c), the Nova Trento granitoid and for the São João Batista granitoid. It is characterized by lack of LREE fractionation relative to $\mathrm{HREE}\left(\mathrm{La}_{\mathrm{n}} / \mathrm{Yb}_{\mathrm{n}}\right.$ ratios variable, between $\left.0.5-4\right)$

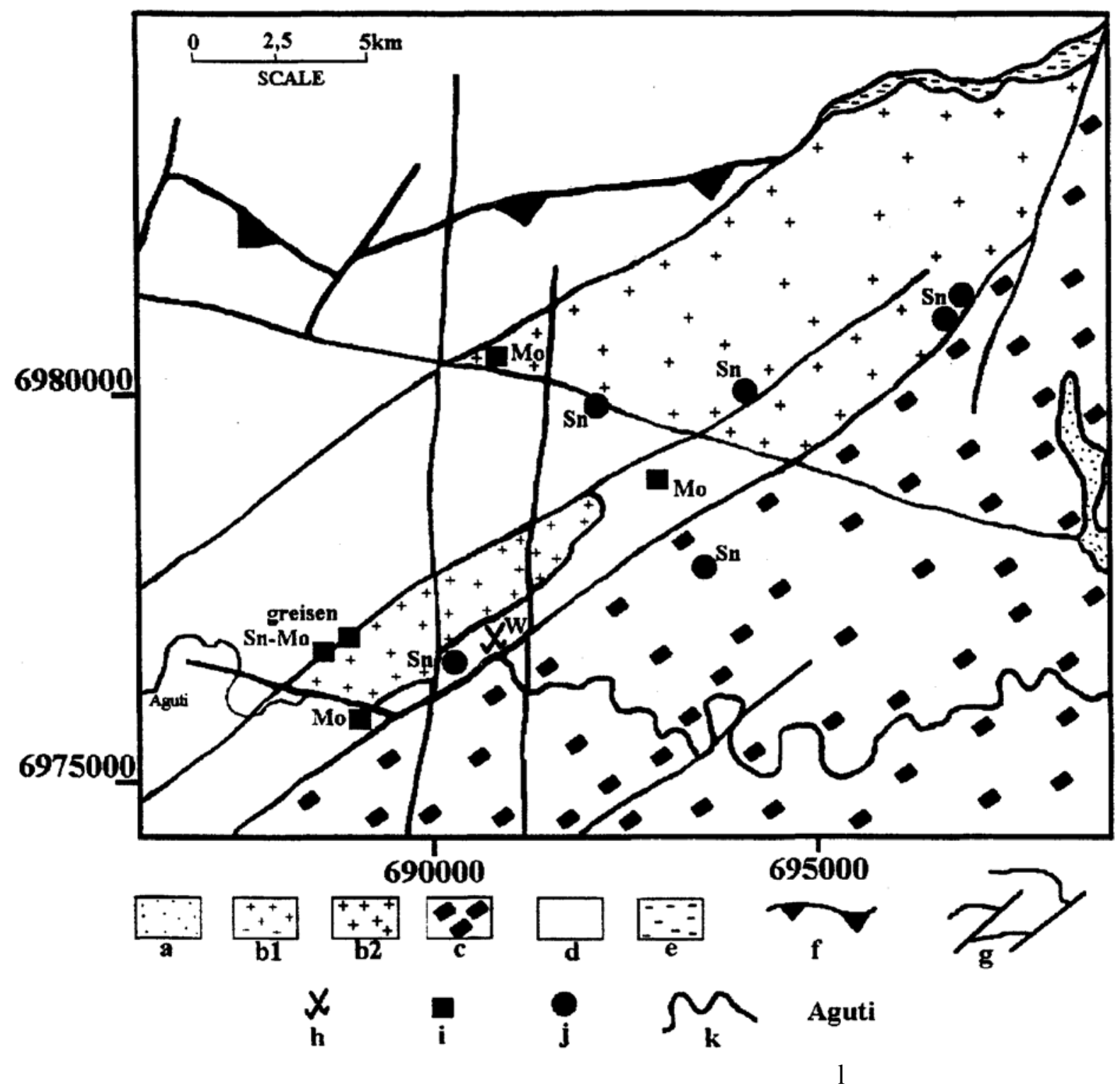

Figure 2 - Major geological features of the Cerro da Catinga mine region, a: Quaternary deposits; b: Catinga Suite: Catinga stock (bl) and Tijucas stock (b2), biotite-muscovite syenogranite and alkali-feldspar granites; c: Valsungana Suite (biotite-quartz-monzonites, monzogranites and minor quartz-gabbros with large microdine crystals); d: Brusque Group (Botuvera Sequence, biotite-muscovite-schists, quartz-schists, quartzites and garnet-muscovite-schists); $\boldsymbol{e}:$ Metamorphic aureole; $f:$ Thrust faults; $\boldsymbol{g}:$ Geologic traces (contacts and faults); h: Abandoned mine of Cerro da Catinga); $\boldsymbol{i}$ : Occurrence of Mo, $W$ and greisen; $\boldsymbol{j}$ : Cassiterite in river sediments; $\boldsymbol{k}$ : River; I: Locality. 
Table 1 - Major petro graphic characteristics of the granitoids intrusive in the Brusque Group.

\begin{tabular}{|c|c|c|c|c|c|c|c|}
\hline $\begin{array}{l}\text { Suites and } \\
\text { granitoids }\end{array}$ & $\begin{array}{c}\text { Macroscopic } \\
\text { characteristics }\end{array}$ & \begin{tabular}{|c|} 
Rock-forming Minerals \\
$(\%)$
\end{tabular} & $\begin{array}{l}\text { accessory } \\
\text { Minerals }\end{array}$ & $\begin{array}{c}\text { Late-magmatic } \\
\text { minerals }\end{array}$ & $\begin{array}{c}\text { Textural } \\
\text { characteristics }\end{array}$ & Structures & Classification \\
\hline $\begin{array}{l}\text { Catinga Suite } \\
\text { Granitoids: Catinga } \\
\text { and Tijucas }\end{array}$ & $\begin{array}{l}\text { White-grayish, } \\
\text { equigranular, medium- } \\
\text { to line-grained } \\
\text { granitoids }\end{array}$ & $\begin{array}{l}\text { quatry (20-35), microcline (10- } \\
54) \text {, albitc (12-46), oligoclase } \\
\text { (5-18), brown-red biolites (TR- } \\
\text { 3), muscovite (TR-5) }\end{array}$ & $\begin{array}{l}\text { zircon, apatitc, } \\
\text { opaque } \\
\text { mincrals, } \\
\text { allanice, } \\
\text { (garrect?) }\end{array}$ & $\begin{array}{c}\text { (cpidole/ } \\
\text { clinozoisite), clay } \\
\text { mincrals, linc- } \\
\text { grained opaque } \\
\text { mincrals, } \\
\text { muscovite/sericite, } \\
\text { chloritc, lluoritc, } \\
\text { (carbonatc), } \\
\text { wolframile? }\end{array}$ & $\begin{array}{l}\text { Equigranular, rarely } \\
\text { incquigranular } \\
\text { hipidiomurjhic }\end{array}$ & $\begin{array}{l}\text { Only brittle structures, } \\
\text { fractures }\end{array}$ & $\begin{array}{l}\text { (biotitc) - muscovitc- } \\
\text { sycnogranitcs and } \\
\text { variations to alkali- } \\
\text { feldspar granites and } \\
\text { quatiz-syenites }\end{array}$ \\
\hline $\begin{array}{c}\text { Faxinal Suite } \\
\text { Faxinal granitoid (F) } \\
\text { Gaspar granitoid } \\
\text { (G) }\end{array}$ & $\begin{array}{l}\text { F: gray-b]ack matrix } \\
\text { with pink K-leldspar } \\
\text { and medium- lo coarsc- } \\
\text { graincd ( } \mathrm{K} \text {-ficldspar } \\
\text { around } 2 \mathrm{~cm} \text { ) } \\
\text { G: brown-red, whitc } \\
\text { (quartz), medium- to } \\
\text { line grained }\end{array}$ & 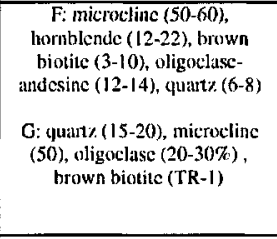 & \begin{tabular}{|} 
F: titanitc \\
(1\%), \\
opaque \\
minerals, \\
apatice, zircon \\
G: opaque \\
mineruls, \\
gircon, apatitc
\end{tabular} & $\begin{array}{c}\text { F: chlorite, fine } \\
\text { opaque mincrals, } \\
\text { scricite. epidiste, } \\
\text { carbonate and clay } \\
\text { minerals } \\
\text { G: scricitcl } \\
\text { muscovite, fluorile, } \\
\text { clay minerals and } \\
\text { line-grained opaque } \\
\text { mincrals } \\
\end{array}$ & $\begin{array}{l}\text { F: incquigranular } \\
\text { hipidionorphic } \\
\text { G: equigranular } \\
\text { hipidiomorphic wo } \\
\text { xenumorphic }\end{array}$ & $\begin{array}{l}\text { F: weak foliation with biotitc, } \\
\text { hornblende and largc K-feldspar } \\
\text { crystals } \\
\text { G: Folintion with biotitc, quart\%, } \\
\text { scricitc/muscovite, locally } \\
\text { cataclastic }\end{array}$ & $\begin{array}{l}\text { F: titanitc-biolitc- } \\
\text { hornblende- quart\%- } \\
\text { syenitc } \\
\text { G: ((biotitc)) - quatit- } \\
\text { monzonitc to } \\
\text { syenogrante }\end{array}$ \\
\hline $\begin{array}{l}\text { Valsungana } \\
\text { Suite }\end{array}$ & $\begin{array}{l}\text { Gray, with large K- } \\
\text { feldspar megacrysus (up } \\
\text { to } 5 \mathrm{~cm} \text { ) in a coarse- } \\
\text { grained matrix }\end{array}$ & $\begin{array}{l}\text { quartz (10-20), microcline } \\
\text { (20-50), oligeclase-andesine } \\
(25-55) \text {, brown-red biotite }(5 \text { - } \\
12 \%) \text {, hornblende (TR) }\end{array}$ & $\begin{array}{c}\text { zircon, } \\
\text { allanic., } \\
\text { litanite, } \\
\text { opaque } \\
\text { minerals, } \\
\text { apatite, } \\
\text { (tourmalinc) } \\
\end{array}$ & $\begin{array}{l}\text { chloritc, finc- } \\
\text { grained opacue } \\
\text { minerals, epidute, } \\
\text { scricite, cliy } \\
\text { mincrals, (lluoritc) } \\
\text { und (caitonatc) }\end{array}$ & $\begin{array}{c}\text { Generally } \\
\text { inequigranular } \\
\text { hipidiomorphic }\end{array}$ & $\begin{array}{l}\text { Foliation. locally cataclastic, } \\
\text { with biotite, some quants and } \\
\text { reoricnted large K-feldspar } \\
\text { crystals }\end{array}$ & $\begin{array}{l}\text { (hornblende)-biolite - } \\
\text { quarz-monzonilc to } \\
\text { monzogranites, quart:- } \\
\text { monzodiorics and } \\
\text { quarty-monzogabbros }\end{array}$ \\
\hline $\begin{array}{c}\text { Morro Pelado } \\
\text { Suite granitoids: } \\
\text { Morro Pelado, Rio } \\
\text { do Alho, Beija Flor, } \\
\text { Santo Aleixo, } \\
\text { Nova Itália, Morro } \\
\text { do Boi and quartz- } \\
\text { feldspatic } \\
\text { mobilizates } \\
\end{array}$ & $\begin{array}{l}\text { Gray and dark gray } \\
\text { sometimes pinkish. } \\
\text { Medium-to line } \\
\text { grained (rare K- } \\
\text { Seldspar up w lcm) }\end{array}$ & $\begin{array}{l}\text { quarty (16-35), microcline (29- } \\
65), \\
\text { albite }(0-16) \\
\text { oligoclase (10)-25), brown-red } \\
\text { biotite(1-12), muscovile(TR- } \\
3 \text { ) }\end{array}$ & \begin{tabular}{|c|} 
ipatite, \\
opaque \\
mincrals, \\
zirron. \\
Lurmaline \\
(around 4 z in \\
the Rio do \\
Alho \\
granitoid) \\
\end{tabular} & $\begin{array}{l}\text { muscovitc/sericite, } \\
\text { epidote /clinozoisite, } \\
\text { chloritc, finc-grained } \\
\text { opaque minerals, } \\
\text { nuorite and clay } \\
\text { minerals }\end{array}$ & $\begin{array}{c}\text { gencrally } \\
\text { equigranular } \\
\text { hipidiomorphic }\end{array}$ & $\begin{array}{l}\text { Foliation with biotilc in the most } \\
\text { samples, rarely line films with } \\
\text { scricite and muscovitc }\end{array}$ & $\begin{array}{c}\text { tourmaline-muscovitc- } \\
\text { biotite-syenogranites } \\
\text { and some variations to } \\
\text { quantz-syenites and } \\
\text { monzogranites }\end{array}$ \\
\hline $\begin{array}{l}\text { Guabiruba } \\
\text { Suite }\end{array}$ & $\begin{array}{c}\text { Pink K-feldspars up to } \\
4 \mathrm{~cm} \text { on the medium to } \\
\text { coarsc-graincd gray } \\
\text { matrix }\end{array}$ & $\begin{array}{c}\text { Quartz }(10-16), \\
\text { microcline }(40-42), \\
\text { oligoclasc-andesine }(30-40), \\
\text { brown to rare } \\
\text { green biotite }(2-3) \\
\text { muscovite }(0-1) \\
\end{array}$ & $\begin{array}{l}\text { allantitc, } \\
\text { titanite, } \\
\text { zircon, } \\
\text { apatile. } \\
\text { opaque } \\
\text { mincriuls } \\
\end{array}$ & $\begin{array}{l}\text { epidorc/cliscroisice, } \\
\text { chloritc, } \\
\text { scricitc, } \\
\text { (carbonate), } \\
\text { clay minerals }\end{array}$ & inequigrannlar & $\begin{array}{l}\text { Incipient foliation with biotite } \\
\text { and some Jeldspar }\end{array}$ & $\begin{array}{l}\text { (muscovitc)-biotite- } \\
\text { quarty-mon\%onitcs }\end{array}$ \\
\hline $\begin{array}{l}\text { Nova Trento } \\
\text { granitoid }\end{array}$ & $\begin{array}{l}\text { granitoids with sume } \\
\text { pink K-lictspars } \\
\text { around } 2 \mathrm{~cm} \text { in a } \\
\text { medium grained gray } \\
\text { matrix; sometimes line } \\
\text { facies with pink tones } \\
\text { occur } \\
\end{array}$ & $\begin{array}{l}\text { quanz (26-35), microcline }(0- \\
52), \text { albitc }(20-4 !), \text { oligoclase } \\
\text { (6-38), brown biotite (TR-6), } \\
\text { muscovile (TR) }\end{array}$ & $\begin{array}{l}\text { (titanite), } \\
\text { allianite, } \\
\text { opaque } \\
\text { minerals, } \\
\text { zircon, } \\
\text { Lourmaline }\end{array}$ & $\begin{array}{l}\text { chlorice, line opaque } \\
\text { mincrals, sericite. } \\
\text { clay minerals. } \\
\text { Duorine, epidusc. } \\
\text { (calbonate) }\end{array}$ & $\begin{array}{l}\text { inequigranular } \\
\text { hipidiomnorphic }\end{array}$ & $\begin{array}{l}\text { Weak loliation with biotitc, } \\
\text { some quartz and reoricnied K- } \\
\text { feldspars }\end{array}$ & $\begin{array}{l}\text { (tourmaline) - biotite - } \\
\text { sycnogranitcs with the } \\
\text { variation to alkali- } \\
\text { feldspar granitc }\end{array}$ \\
\hline $\begin{array}{l}\text { São João Batista } \\
\text { granitoid }\end{array}$ & $\begin{array}{l}\text { Whitc-grayish, medium } \\
\text { to fine grained } \\
\text { granitoids }\end{array}$ & $\begin{array}{l}\text { quatt: (22-32), microcline (20) } \\
59) \text {, albitc (0-48), oligoclase } \\
(2-26) \text {, brown-reddish biotice } \\
\text { (TR-1), muscovitc (TR-7) }\end{array}$ & $\begin{array}{c}\text { ippalite, } \\
\text { opacuec } \\
\text { mincrals, } \\
\text { zircon, } \\
\text { (tourmaline) } \\
\end{array}$ & $\begin{array}{l}\text { Clay minerals, line } \\
\text { opaque mincrals, } \\
\text { 1]uorinc, sericite and } \\
\text { chlorite }\end{array}$ & $\begin{array}{c}\text { equi to } \\
\text { incquigranular } \\
\text { hipidiomorphic }\end{array}$ & Only fructures & $\begin{array}{c}\text { biotitc - muscovite - } \\
\text { alkali-feldspar granitc } \\
\text { with variations to } \\
\text { monzo/syenogranite }\end{array}$ \\
\hline $\begin{array}{l}\text { Campo Novo } \\
\text { Segundo } \\
\text { granitoid }\end{array}$ & $\begin{array}{l}\text { Gray-pink wones, } \\
\text { medium grained }\end{array}$ & $\begin{array}{l}\text { quatz (18), microeline (54), } \\
\text { oligoclasc-andesine (20), } \\
\text { brown biotite (4), } \\
\text { hormblende(2) }\end{array}$ & $\begin{array}{c}\text { títanite, } \\
\text { apatitc, xircon, } \\
\text { opaque } \\
\text { minerals, } \\
\text { allanile } \\
\end{array}$ & $\begin{array}{l}\text { Fine opaque } \\
\text { minerals, chlorite. } \\
\text { clay-minerals. } \\
\text { sericite, cpicluce }\end{array}$ & $\begin{array}{c}\text { cqui a inequigranular } \\
\text { hipidiomorphic }\end{array}$ & $\begin{array}{c}\text { Foliation with biotitc, } \\
\text { homblende, quarty and major } \\
\text { reldspars, locally with cataclastic } \\
\text { characteristics }\end{array}$ & $\begin{array}{l}\text { (titanitc)-hornblende - } \\
\text { biotite - quarty-syenitc }\end{array}$ \\
\hline
\end{tabular}

and a strong negative Eu anomaly. The São João Batista granitoid presents a hybrid REE distribution pattern in relation to both types mentioned above, with high LREE fractionation relative to the HREE $\left(\mathrm{La}_{\mathrm{n}} / \mathrm{Yb}_{\mathrm{n}} \approx 63\right)$ and a pronounced negative Eu anomaly.

In the alumina/alkalis $v s$. alumina/(alkalis $+\mathrm{CaO})$ molecular proportions diagram (Fig. 8) the majority of the granitoids plot in the field of metaluminous rocks. The plutons plot in the boundary between the metaluminous and peraluminous fields. The Faxinai granitoid in the northern domain, is the only pluton that present mesonormative hornblende; the other plutons, including the Ribeirão das Velhas sub-volcanic rocks, on the other hand, present mesonormative corundum (Table 3).

Figure 9 is the La Roche's et al. (1980) $\mathrm{R}_{1}$ vs. $\mathrm{R}_{2}$ diagram with Batchellor \& Bowden's (1985) petrotectonic fields. In this diagram, the great majority of studied granitoids plot in field 4 (late-orogenic), but the Catinga granitoids are transitional between the fields 5 and 7 (anorogenic and post-orogenic, respectively). The more basic fades of the Faxinai pluton (northern domain) plots in the field 3 (post-collision uplift) and even above the 1:1 line, thus characterizing a distinct behavior relative to other plutons in the Brusque Group.

A strong continental crust involvement in the generation of the granitoids under consideration can be depicted by observing their behavior in the diagrams proposed by Pearce et al. (1984), Harris et al. (1986) and Whalen etal. (1987) (respectively Figs. 10,11 and 12). The Catinga granitoids (black field), unlike the other granitoids in the region, plot in the within-plate granite, Fig. 10), transitionally between ii (syn-collisional) and iii (late to after collision alkali-calcic, Fig. 11) and A (A-type, Fig. 12) fields.

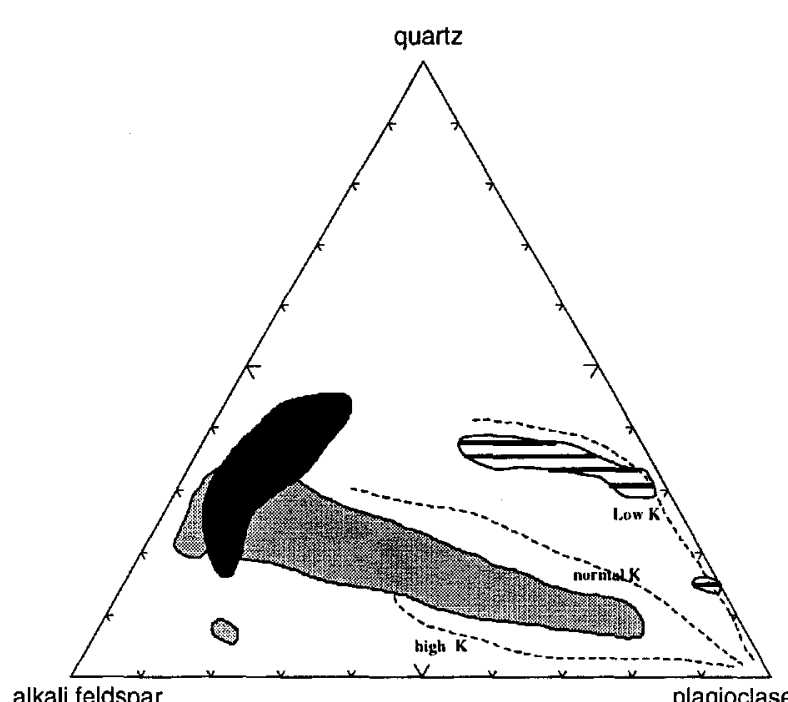

alkali feldspar

plagioclase

Figure 3 - QAP diagram (Streckeisen 1976) for the granitoids intrusive in the Brusque Group. The low K, normal $K$ and high $K$ trends are from Lameyre \& Bowden (1982). Fields: black (Catinga Suite, 16 samples), gray (other granitoids and Ribeirão da Velha sub-volcanic rocks), horizontal lines (gneiss enclaves and Ponta do Cabeço gneisses). 
Table 2 - Whole-rock chemical analyses for the granitoids intrusive in the Brusque Group. References are: open = Open University laboratories (major elements by XRF; trace and RE elements by INAA). keele $=$ Keele University laboratories (trace elements by XRF). act $=$ Actlabs $($ major and trace elements by ICP-AES; trace elements by XRF; trace and RE elements by INAA). geosol = Geosol laboratories (REE by ICP-AES). Basel (1985) = sample from Basel (1985).

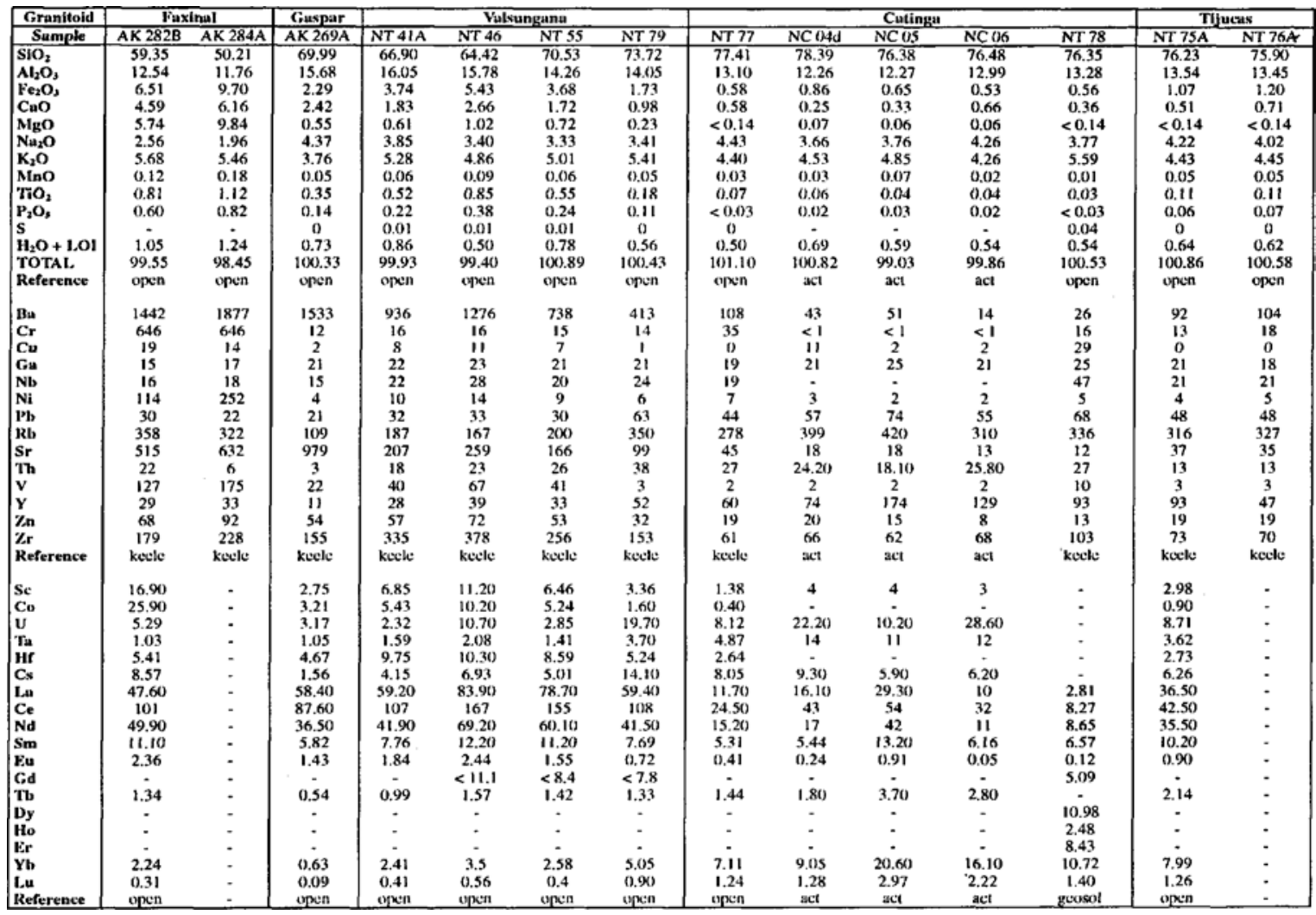

\begin{tabular}{|c|c|c|c|c|c|c|c|c|c|c|c|c|}
\hline Grunitoid & R. Alho & $\begin{array}{c}\text { M. } \\
\text { peludo }\end{array}$ & N. Itailiti & M. Boi & $\begin{array}{c}\text { Ptal. } \\
\text { Callecoso }\end{array}$ & & S.I. Bati & & $\underset{\text { Trento }}{\mathrm{N} .}$ & Rilhelr & da Velha S & volcanics \\
\hline Sample & NT 58 & NT 67 & CAN 37 & \begin{tabular}{|c|} 
M. $\mathrm{BOl}$ \\
2 \\
\end{tabular} & $\begin{array}{c}\text { PTA } \\
\text { CABECO } \\
\end{array}$ & NT 85 & NT 82 & NT 84 & NC 07a & NT 33 & NT 287.7 & NT 286.7 \\
\hline $\mathrm{SlO}_{2}$ & 73.54 & 72.21 & 70.30 & 74.29 & 73.62 & 74.22 & 72.62 & 71.11 & 76.55 & 67.66 & 67.77 & 68.33 \\
\hline $\mathbf{A l}_{2} \mathbf{O}_{3}$ & 14.39 & 14.12 & 14 & 13.76 & (13.69) & 13.52 & 15.21 & 15.77 & 12.74 & 14.59 & 14.62 & 14.88 \\
\hline $\mathrm{Fe}_{2} \mathrm{O}_{3}$ & 186 & 1.91 & 2.62 & 1.66 & 1.98 & 1.73 & 0.33 & 0.56 & 1.52 & 2.18 & 2.64 & 2.41 \\
\hline $\mathrm{CuO}$ & 1.08 & 1.30 & 1.72 & 1.41 & 1.49 & 0.88 & 0.77 & 0.75 & 0.90 & 1.29 & 1.03 & 0.85 \\
\hline MgO & 0.39 & 0.25 & 0.63 & 0.40 & 0.51 & 0.18 & $<0.14$ & 0.15 & 0,15 & 1.45 & 1.83 & 1.72 \\
\hline $\mathrm{Na}_{2} \mathrm{O}$ & 3.40 & 3.80 & 3.07 & 3.38 & 3.37 & 3.66 & 5.28 & 4.93 & 3.70 & 2.36 & 2.35 & 2.39 \\
\hline $\mathrm{K}_{2} \mathrm{O}$ & 5.20 & 5.22 & 4.98 & 4.73 & 4.88 & 4.86 & 4.88 & 5.50 & 4.47 & 7.35 & 6.95 & 7.24 \\
\hline Mno & 0.05 & 0.04 & 0.04 & 0.04 & 0.05 & 0.08 & 0.01 & 0.01 & 0,05 & 0.05 & 0.07 & 0.05 \\
\hline $\mathrm{riO}_{2}$ & 0.24 & 0.23 & 0.42 & 0.18 & 0.22 & 0.22 & 0.14 & 0.22 & 0.12 & 0.51 & 0.57 & 0.57 \\
\hline $\mathrm{P}_{2} \mathrm{O}_{5}$ & 0.06 & 0.10 & 0.09 & 0.07 & 0.07 & 0.09 & 0.05 & 0.08 & 0,04 & 0.35 & 0.44 & 0.39 \\
\hline $\mathbf{s}$ & 0.01 & 0 & & & & () & 0.01 & 0.01 & & & 0.01 & 0 \\
\hline $\mathrm{H}_{2} \mathrm{O}+\mathrm{LOI}$ & 0.58 & 0.74 & 0.73 & 0.49 & 0.74 & 0.88 & 0.70 & 0.74 & 0,59 & 0.67 & 0.72 & 0.87 \\
\hline TOTAL & 100.80 & 99.92 & 28.60) & 100.41 & $1(0) .62$ & 100.32 & 100 & 99.83 & $1(x) .83$ & 98.46 & 99 & 99.70 \\
\hline Reference & open & open & $\begin{array}{c}\text { Basci } \\
\text { (1985) }\end{array}$ & act & act & wct & $(n) \times n$ & 1) & act & upen & open & open \\
\hline Ba & - & 780 & $129 \%$ & 1769 & 841 & 359 & 339 & 492 & 116 & 1183 & 733 & 748 \\
\hline Cr & : & 15 & - & 8 & 12 & 13 & 18 & 19 & $<1$ & 69 & 83 & 77 \\
\hline $\mathrm{Cu}$ & - & 3 & - & 2 & 2 & 2 & () & i & 3 & 5 & 13 & 9 \\
\hline Ga & - & 18 & 20) & 18 & 17 & 19 & 39 & 35 & 24 & 25 & 28 & 30 \\
\hline $\mathbf{N i}$ & - & 12 & 26 & 16 & 14 & 24 & 14 & 16) & 28 & 42 & 44 & 44 \\
\hline Ni & - & 4 & 3 & 4 & 6 & 8 & 6 & 5 & 3 & 38 & 42 & 19 \\
\hline 16 & - & 26 & 29 & 40 & 33 & 50 & 48 & 66 & 47 & 107 & 153 & 381 \\
\hline Rl, & - & 241 & 168 & 128 & 180 & 321 & 453 & 497 & 261 & 411 & 413 & 440 \\
\hline $\mathbf{S r}$ & - & 183 & 220 & 312 & 260 & 88 & 134 & 165 & 39 & 372 & 322 & 319 \\
\hline Th & 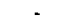 & 15 & 39 & 18.30 & 25.50 & 28 & 29 & 39 & 35.80 & 62 & 62 & 64 \\
\hline$v$ & - & 15 & 20 & 10) & 10) & 19 & 8 & 9 & 2 & 22 & 44 & 24 \\
\hline $\mathrm{Y}$ & - & 26 & 42 & 15 & 9 & 190 & 8 & 7 & 51 & 16 & 18 & 19 \\
\hline$Z_{n}$ & - & 37 & 31 & 27 & 37 & 44 & 17 & 59 & 33 & 71 & 67 & 45 \\
\hline & . & 195 & 279 & 167 & 144 & 119 & 109 & 158 & 125 & 432 & 444 & 464 \\
\hline Reference & - & kwele & Basei & act & act & keele & kecte & kecelc & aci & kecile & kecle & kete \\
\hline Se & - & - & - & 3 & 3 & 3.75 & 1.44 & 1.42 & 4 & . & 6.29 & . \\
\hline Co & & & & & 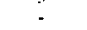 & 1.94 & 0.70 & 0.81 & 7 & . & 7.44 & . \\
\hline v & - & - & 13 & 1.80 & 3.90 & 9.39 & 23.80 & 21 & 17.60 & - & 31.30 & - \\
\hline Tw & 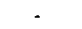 & 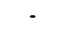 & : & 3.10 & 2.50 & 3.30 & 1.80 & 0.92 & 5.40 & - & 6.74 & - \\
\hline Hr & - & - & - & & & 3.79 & 4.31 & 5.66 & - & - & 15.20 & 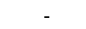 \\
\hline C.s & & & & 2.7 & 4 & 10.50 & 21.10 & 24.50 & 6 & 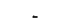 & 16.40 & - \\
\hline 1.4 & - & 56.76 & 125 & 78.40 & 59.90 & 73.40 & 30) & 47,40 & 26,90 & 67.43 & 102 & 96.25 \\
\hline $\mathrm{Ce}$ & - & 117.20 & 195 & 121 & 1106 & 65.30 & 60.50 & 89,40 & 56 & 153,70 & 208 & 218.40 \\
\hline Nd & - & 39,86 & & 35 & 34 & 85 & 29 & 37.60 & 24 & 66.21 & 87.70 & $90.60)$ \\
\hline $\mathbf{S}_{\mathbf{m}}$ & 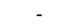 & 6.95 & - & 4.74 & 4.55 & 23 & 4.45 & 5.61 & 5.60 & 11.22 & 13.20 & is \\
\hline Eu & . & 0.95 & - & 0.87 & 0.92 & 3.11 & 0.49 & 0.71 & 0.52 & 1.37 & 1.37 & 1.66 \\
\hline Gd & 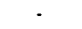 & 4.42 & & & & $<31,9$ & & & & 5.02 & $<14$ & 6.61 \\
\hline $\mathrm{Tb}$ & - & - & - & 0.30 & 0.20 & 5.66 & 0.31 & 0.51 & 1.80 & - & 1.33 & \\
\hline Dy & - & 3.68 & 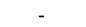 & 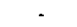 & 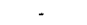 & 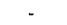 & 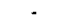 & 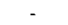 & 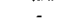 & 2,71 & . & 3.69 \\
\hline $\mathrm{Ho}$ & - & 0.71 & - & - & . & - & - & - & - & 0.49 & - & 0.66 \\
\hline $\mathrm{Er}$ & - & 2.01 & - & - & - & - & - & - & - & 1.08 & - & 1.37 \\
\hline Yb & & 1.76 & - & 1.43 & 0.96 & 18.80 & 0.35 & 0.33 & 5.15 & 1).79 & 0.97 & 1.05 \\
\hline & - & 0.25 & - & 0.22 & 0.14 & 3.15 & $<0,08$ & - & 18.79 & 0.12 & & 0.13 \\
\hline Reference & 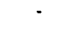 & geosul & $\begin{array}{l}\text { Basei } \\
1985\end{array}$ & act & act & copen & oncin & "1p:-1 & wat & geosol & 2 & geosol \\
\hline
\end{tabular}


The comparison between the studied granitoids with Sn-mineralized granitoids from other regions, in terms of $\mathrm{Rb}, \mathrm{Sr}, \mathrm{Ba}$ and $\mathrm{K}, \mathrm{O}$ contents, is represented in Figs. 13 and 14. Once these elements are found in the main mineral phases of the granitoid rocks (feldspars, biotite and hornblende), they can properly act as monitors of fractionation processes and granitoid magma evolution. In this context, the importance of such monitoring is that the concentrations of metals with crustal affinity ( $\mathrm{Sn}, \mathrm{W}, \mathrm{Rb}, \mathrm{Li}$, etc.) plot in the more differentiated and/or evolved portions of certain diagrams. In the $\mathrm{Rb} \times \mathrm{Ba} \times \mathrm{Sr}$ triangular diagram of El Boseily \& El Sokkary (1975; Fig. 13) the less evolved terms of the studied granitoids plot in the field of granitoids with anomalous composition. The more evolved terms, however, plot in the field of highly differentiated granites. The Catinga granitoids are more enriched in $\mathrm{Rb}$, thus plotting in the field of highly differentiated granitoids; overlapping the average for the São Francisco and Água Boa Sn-bearing granitoids as respectively described by Daoud \& Antonietto Jr. (1985) and Pelachin \& Daoud (1988).
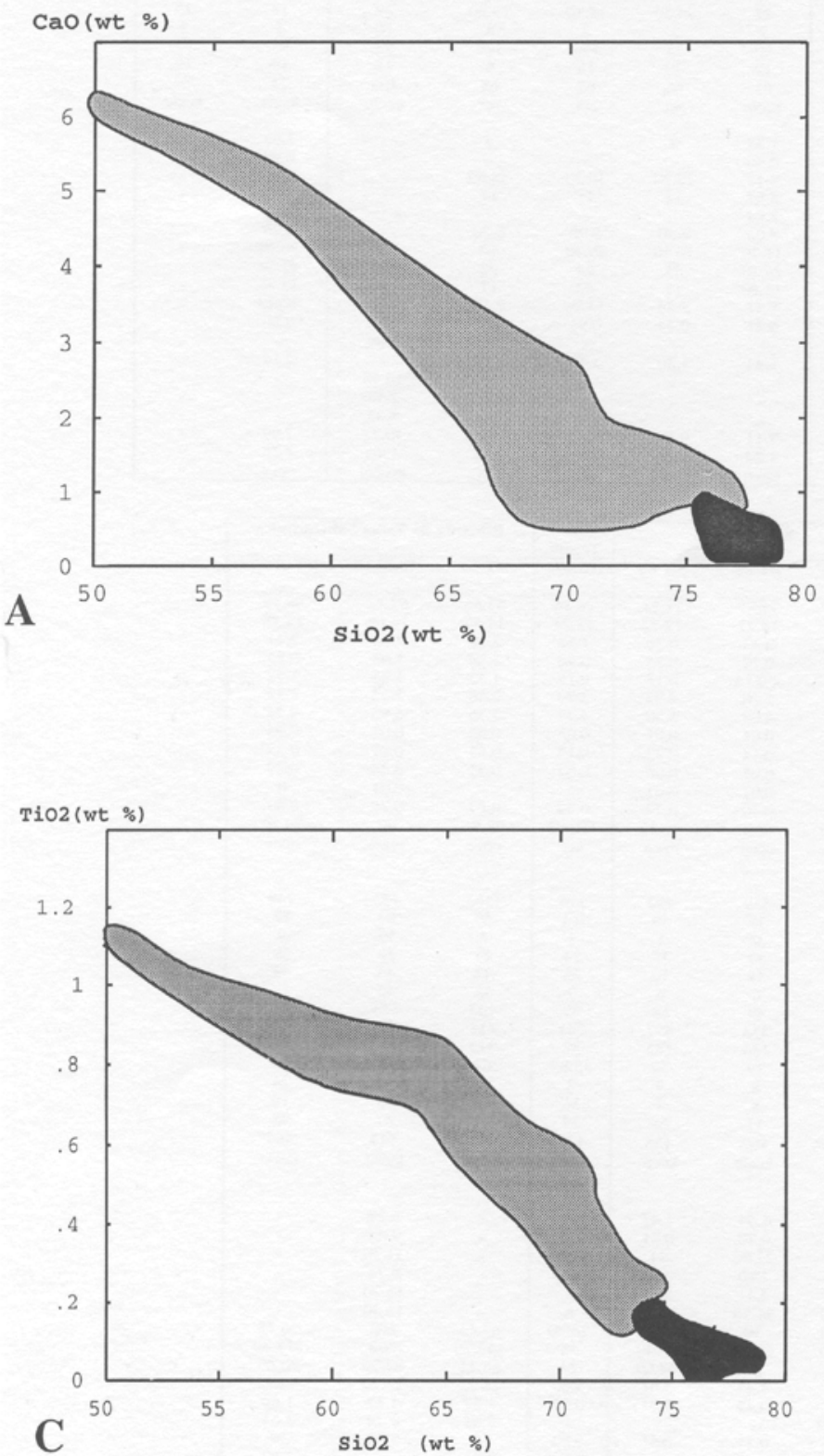

Table 3 - Mesonormative composition of the granitoids intrusive in the Brusque Group and Ribeirão da Velha sub-volcanic rocks.

\begin{tabular}{|c|c|c|c|c|}
\hline & \# Samples & Corindon (\%) & Hornblende (\%) & Biotite (\%) \\
\hline Faxinal granitoid & 2 & 0 & 48.4 & 11.1 \\
\hline $\begin{array}{c}\text { Ribeirão da } \\
\text { Velha sub- } \\
\text { volcanic rocks }\end{array}$ & 3 & 2.1 & 0 & 11.4 \\
\hline Valsungana Suite & 3 & 1.5 & 0 & 12.4 \\
\hline Catinga Suite & 6 & 0.9 & 0 & 3.7 \\
\hline $\begin{array}{c}\text { Morro Pelado } \\
\text { Suite }\end{array}$ & 2 & 0.7 & 0 & 5.4 \\
\hline $\begin{array}{c}\text { Nova Trento } \\
\text { granitoid }\end{array}$ & 1 & 0.3 & 0 & 3.9 \\
\hline $\begin{array}{c}\text { São João Batista } \\
\text { granitoid }\end{array}$ & 3 & 0.3 & 0 & 1.2 \\
\hline
\end{tabular}
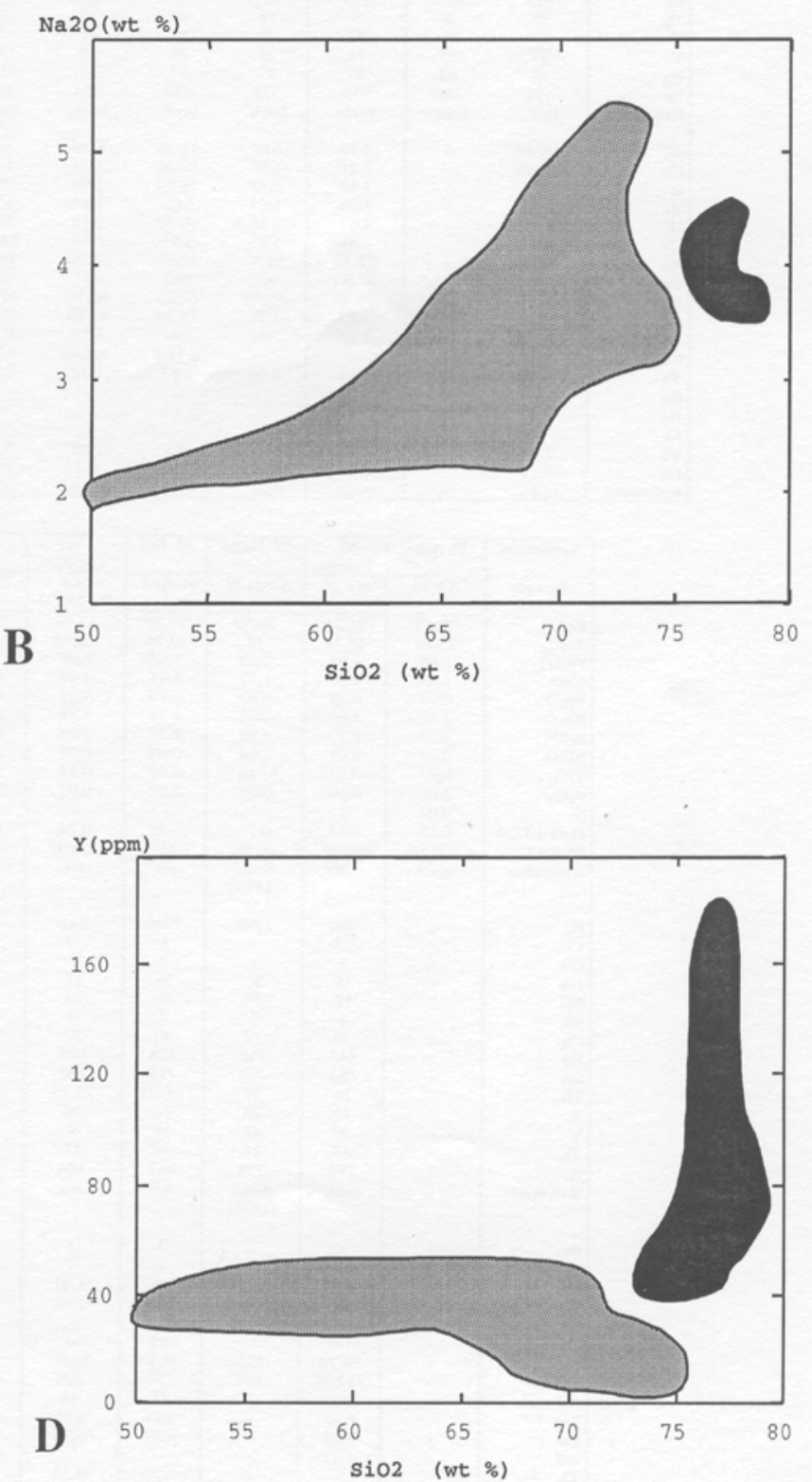

Figure 4 - Main trends in Harker's diagrams (major and trace elements). Black field: Catinga Suite (CS); gray field: other granitoids intrusive in the Brusque Group and Ribeirão da Velha sub-volcanic rocks. Number of samples: 25. 
FAXINAL SUITE
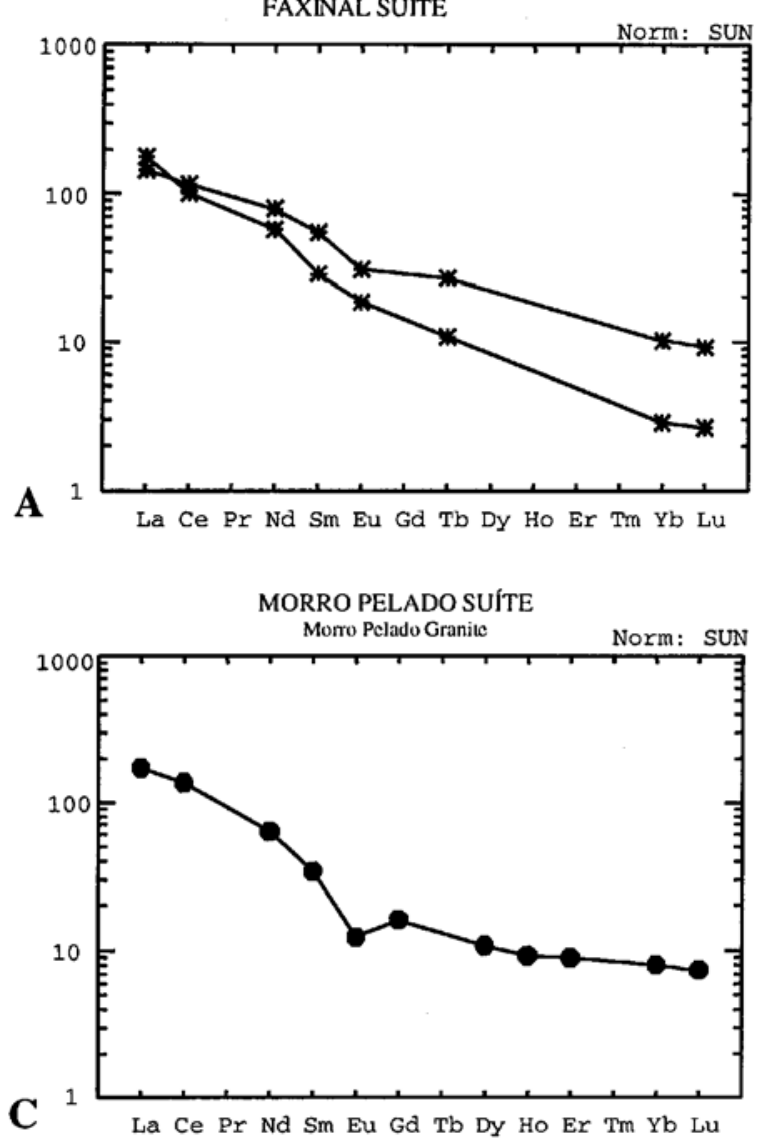

Ribeirão da Velha Sub-Volcanics

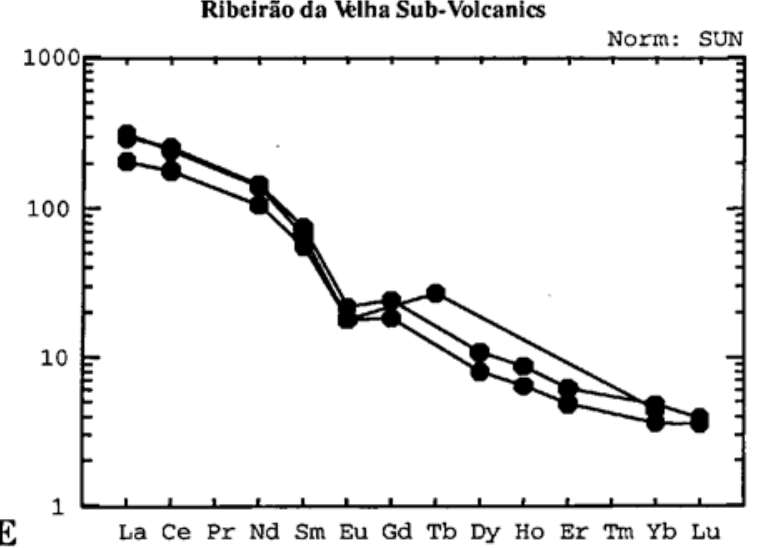

VALSUNGANA SUITE

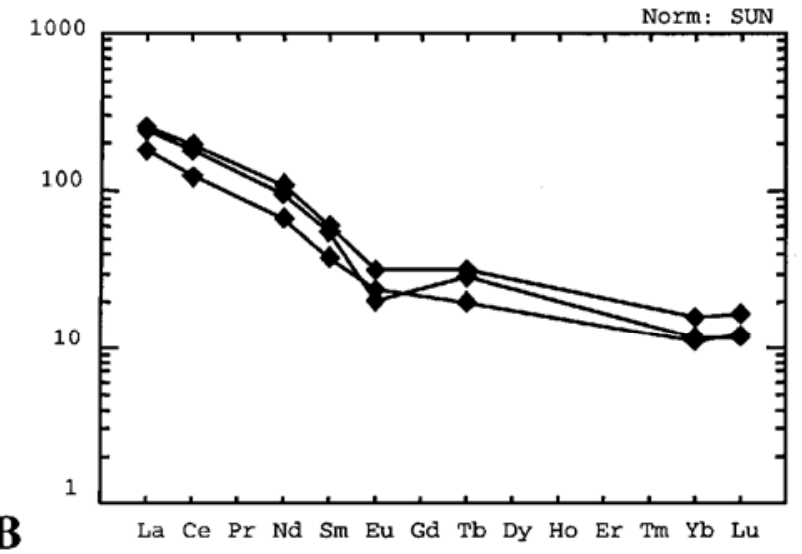

MORRO PELADOSUITE

Nova ltália (open square) and Morro do Boi granitoids

(filled square); quartz-fcldspatic mobilizates of the Ponta Norm: SUN do Cabeço (halr filled squarc)

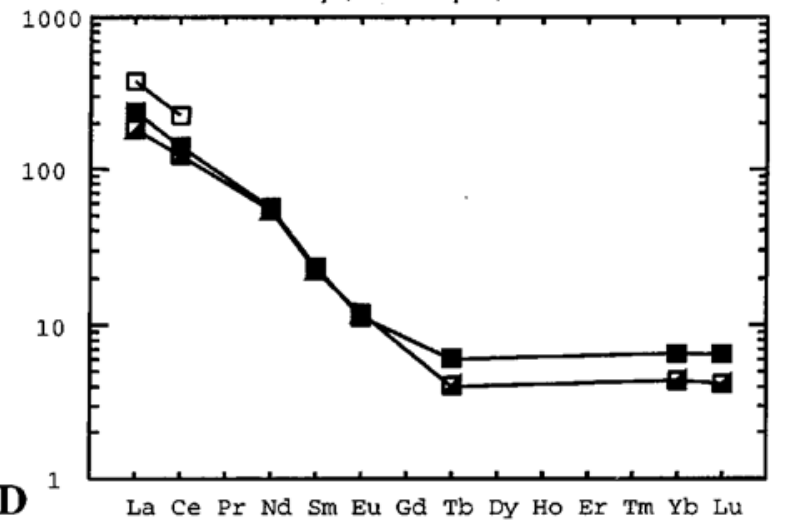

Figure 5 - REE patterns for the Faxinai, Valsungana and Morro Pelado suites, other granitoids and Ribeirão da Velha sub-volcanic rocks. Values for normalization are from Sun (1982).

Figure 14 presents the $\mathrm{K}_{2} \mathrm{O}$, Sr and $\mathrm{Ba} v s$. $\mathrm{Rb}$ diagrams, with the fields proposed by Javier Rios et al. (1995). Samples from the Catinga granitoids plot mostly within the field for Sn-mineralized granitoids, while other granitoids in the region plot away from those fields.

FINAL CONSIDERATIONS The granitoids intrusive in the southern domain of the Brusque Group bear whitish-gray K-feldspar and brown to reddish-brown biotite, which together with negative $\mathrm{Eu}$ anomaly in chondrite-normalized REE diagrams, indicate reduced conditions of magma formation (White 1992; Wilson 1991). Such reducing conditions, high fluorine concentrations as indicated by the presence of fluorite and tourmaline, water-saturated conditions, as suggested by muscovite, and the fact that these are crustal granitoids, greatly contributed to determine the metallogenetic specialization of the studied granitoids. The Catinga granitoids, which are the most evolved ones in the region, is the pluton in which one can find the main concentrations of metals. The petrographic and lithochemical characteristics of these granitoids are compatible with those observed in crustal granitoids that bear metal concentrations, such as $\mathrm{W}, \mathrm{Sn}$, and Mo).

There are several similarities between the Catinga suite and the São João Batista and Nova Trento granitoids, such as presence of alkalic feldspars (albite), reddish-brown biotite, fluorite as late-magmatic mineral, relatively high $\mathrm{Na}_{2} \mathrm{O}$ content, and a weak LREE fractionation relative to the HREE, generally associated with a pronounced negative $\mathrm{Eu}$ anomaly. A crustal source for some of the granitoids intrusive in the southern domain of the Brusque Group is attested by the high initial ${ }^{87} \mathrm{Sr}^{86} \mathrm{Sr}$ ratios (Basei 1985). Sm-Nd isotopic data (Mantovani et al. 1987 ) indicate $t_{\mathrm{DM}}$ model ages around 2,020 Ma which testify the long crustal residence for the majority of the protoliths of the rocks present in the Brusque Group. Gneisses and migmatites that occur in the eastern portion of the Brusque Group are suggested to be possible protoliths for these granitoids. However, the available geochronological data for these gneisses indicate a Rb-Sr age older than that of the crust likely to have originated the Valsungana granitoid $(2,590 \pm 350$ 

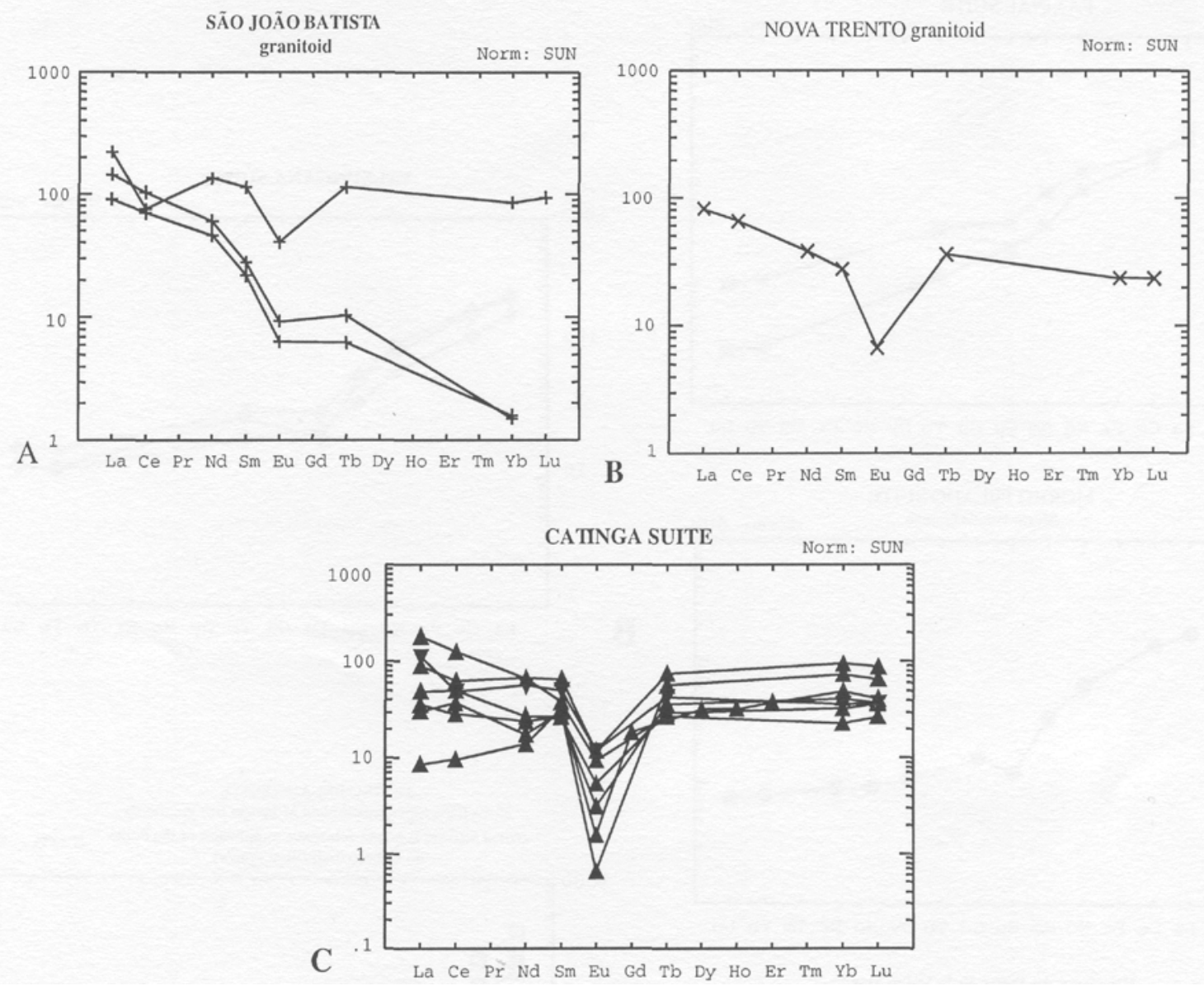

Figure 6 - REE patterns for the Catinga Suite and other granitoids. Values for normalization are from Sun (1982).

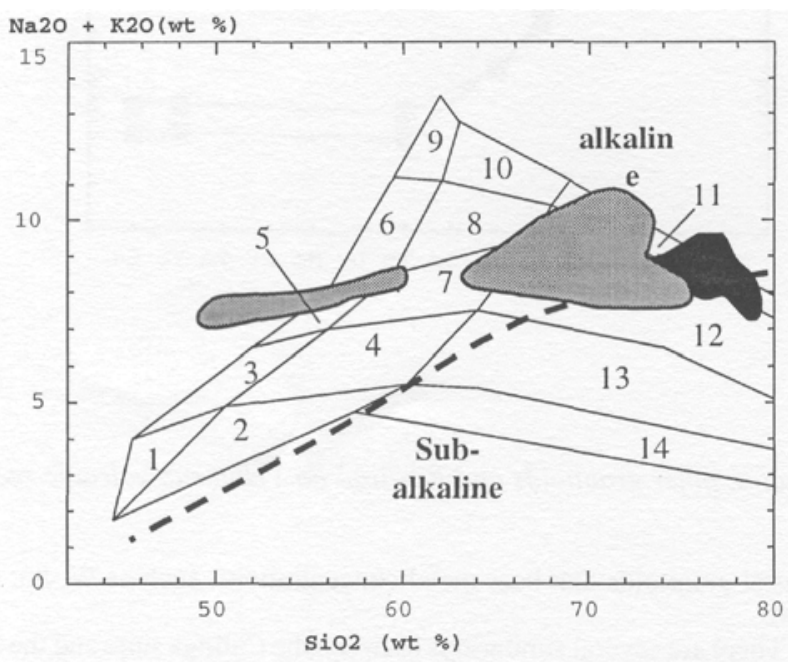

Figure 7 - T AS diagram with lithologic (Middlemost 1985) and alkaline and sub-alkaline fields (Miyashiro 1978). B lack field: Catinga Suite; gray field: other granitoids and Ribeirão da Velha sub-volcanic rocks. Number of samples: 25. Fields: 1-Diorite and gabbro, 2Quartz-diorite, 3-Monzodiorite, 4- Quartz-monzodiorite, 5-Monzonite, 6- Syenite, 7-Quartz-monzonite, 8-Quartz-syenite, 9-Alkalifeldspar syenite, 10-Alkali-feldspar quartz-syenite, 11-Alkali-feldspar granite, 12-Granite, 13-Granodiorite, 14-Tonalite.

$\mathrm{Ma},{ }^{87} \mathrm{Sr} /{ }^{86} \mathrm{Sr} \pm 0.701$; Basei 1985$)$, so that more studies are necessary to confirm this hypothesis.

In the northern domain of the Brusque Group, K-feldspar in the granitoids is predominantly pink, hornblende is conspicuous in the

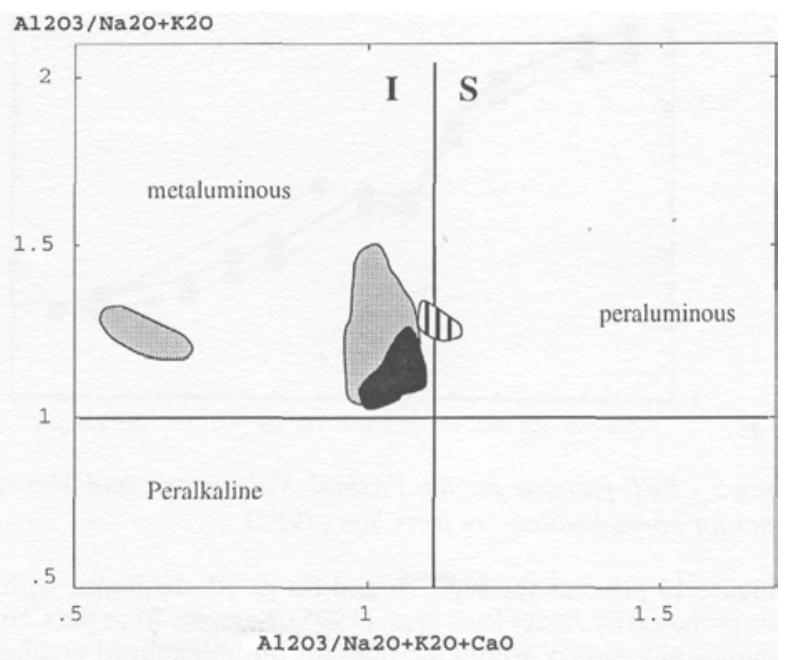

Figure 8 - Alumina/alkalis vs. alumina/(alkalis $+\mathrm{CaO}$ ) (molecular proportions) diagram. Black field: Catinga Suite; Field with vertical lines: Ribeirão da Velha sub-volcanic rocks; Gray field: other granitoids. Number of samples: 25.

more basic compositions, and macroscopic fluorite is observed in pink quartz-feldspathic types. In this region the metal concentrations possibly associated with the granitoids are preferentially auriferous. Incipient negative Eu anomaly in REE patterns for the granitoids, together with presence of slightly pink K-feldspar, absence of reddish biotite in the Guabiruba suite, lack of Sn-bearing granitoids and the presence of a series of $\mathrm{W}$ and $\mathrm{Au}$ occurrences in this domain, suggest a more oxidizing conditions in the generation of the magmas, than for the 


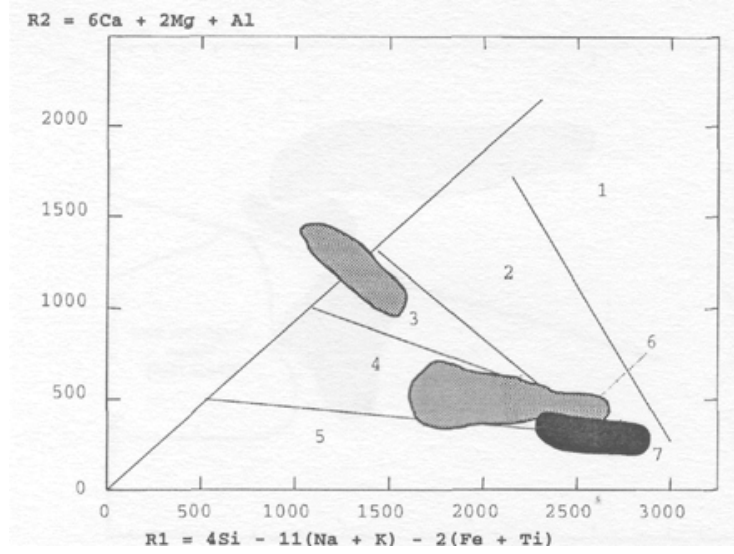

Figure $9-R 1 \times R 2$ cationic diagram from La Roche et al. (1980) with petrotectonic fields from Bachelor \& Bowden (1985). Black field: Catinga Suite; gray field: other granitoids intrusive in the Brusque Group and Ribeirão da Velha sub-volcanic rocks. Number of samples: 23. Fields: 1-Mantle fractionates, 2-Pre-plate collision, 3'-Post-collision uplift, 4-Late-orogenic, 5-Anorogenic, 6-Syn-collision and 7Post-collision.

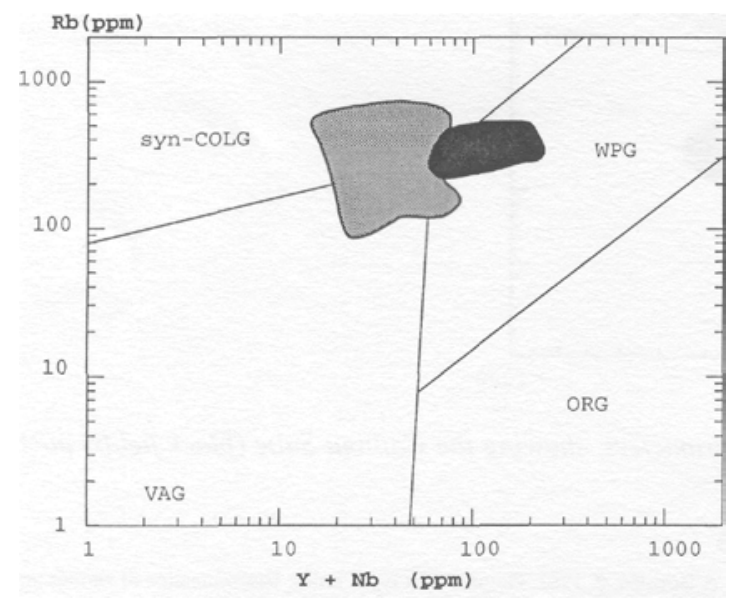

Figure $10-R b \times(Y+N b)$ diagram (discriminant fields after Pearce et ai. 1984). Black field: Catinga Suite; gray field: other granitoids intrusive in the Brusque Group and Ribeirão da Velha sub-volcanic rocks. Number of samples: 23. Fields: VAC: Volcanic arc granites, SYN-COLG: Syn-collision granites, WPG: Within-plate granites and ORG: Ocean ridge granites.

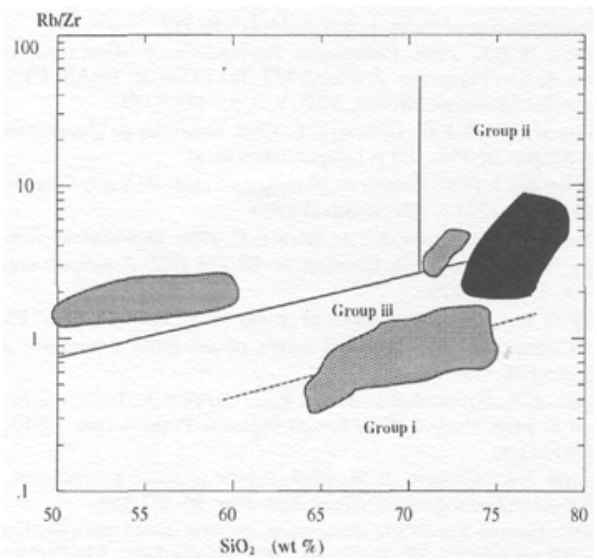

Figure $11-\mathrm{Rb} / \mathrm{Zr} \times \mathrm{SiO}_{2}$ diagram (discriminate fields after Harris et ai. 1986). Black field: Catinga Suite; Gray field: other granitoids intrusive in the Brusque Group and Ribeirão da Velha sub-volcanic rocks. Number of samples: 25. Fields: i (pre-collision volcanic-arc), ii (syn-collision), Hi (late to after collision alkali-calcic).

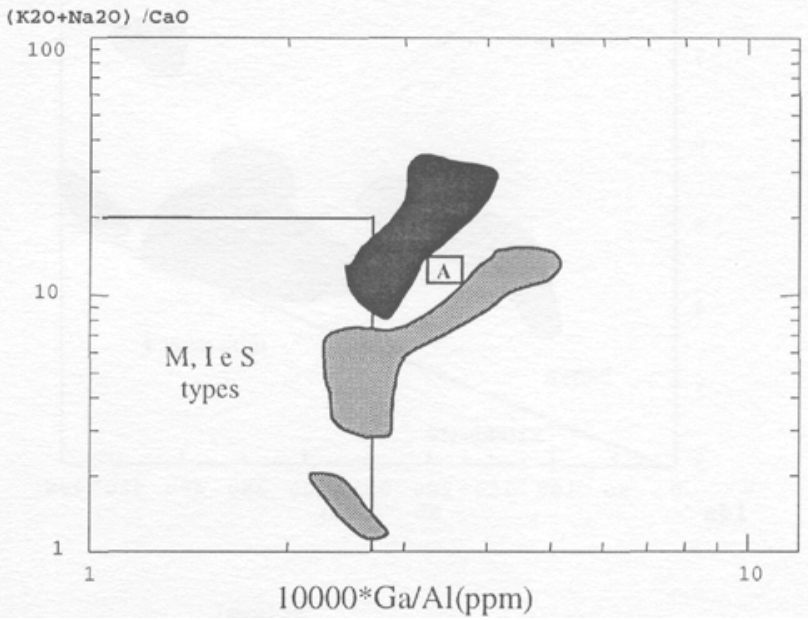

Figure 12 - Alkalis/CaO $\times$ Ga/Al diagram (discrimination fields after Whalen et al. 1987) showing the transitional behavior to A-type granitoids of almost granitoids intrusive in the Brusque Group. Black field: Catinga Suite; gray field: other granitoids and Ribeirão da Velha sub-volcanic rocks. Number of samples: 25.

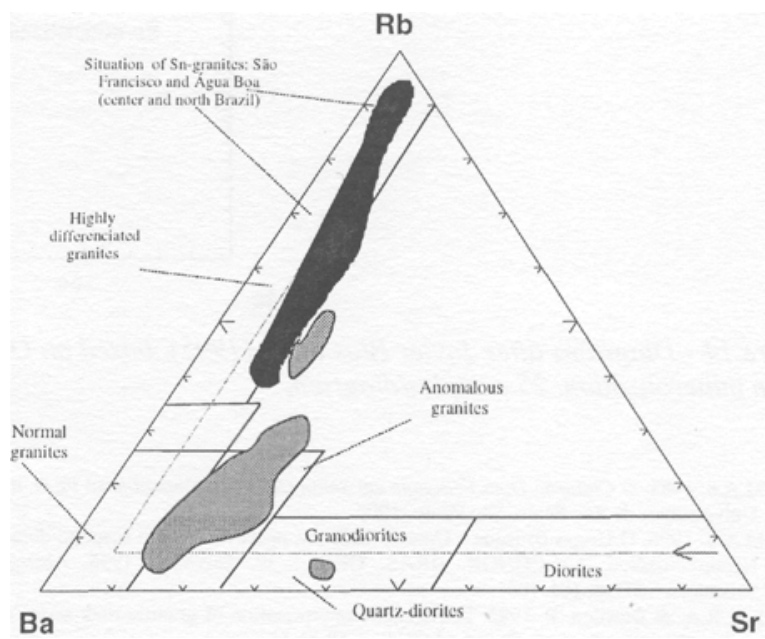

Figure $13-R b, B a$ and $\mathrm{Sr}$ triangular diagram (discrimination fields after El Boseily \& El Sokkary J975). The less differentiated portions oft/re granitoids intrusive in the Brusque Group plot on the anomalous granite fields. The Calinga Suite (black field) shows typical behavior of highly differentiated granites and some tin-bearing granites (data from Daoud \& Antonietto Jr. 1985 and Pelachin \& Daoud 1988). Number of samples: 25.

magmas in the southern domain. The more basic composition of the Faxinai pluton indicates less evolved crustal components.

Basei (1985) postulates for the region a major Neoproterozoic collisional system, with vergence from southeast to northwest, which took place with no influence of an oceanic crust in the active subduction processes, as attested by the presence of large volume of crustal granitoids in the region. Basei (1996) showed that such collisional system is characterized by the allochtonous of huge crustal segments placed between major faults (Ribeirão da Prata Belt and Major Gercino shear zones). It is still to be revealed to which extent the granite genesis in the Brusque Group domain has suffered influence of older continental crust with different $t_{\mathrm{DM}}$ ages.

Acknowledgements To FAPESP (Proc. 94/4136-1 and 93/2627-3) and $\mathrm{CNPq}$ (Proc. 52174695-7) for the financial support; Dr. Valdecir Janasi from IG-USP for the valuable discussions, and to the anonymous reviewers of RBG for the suggestions which greatly improved the original text. 

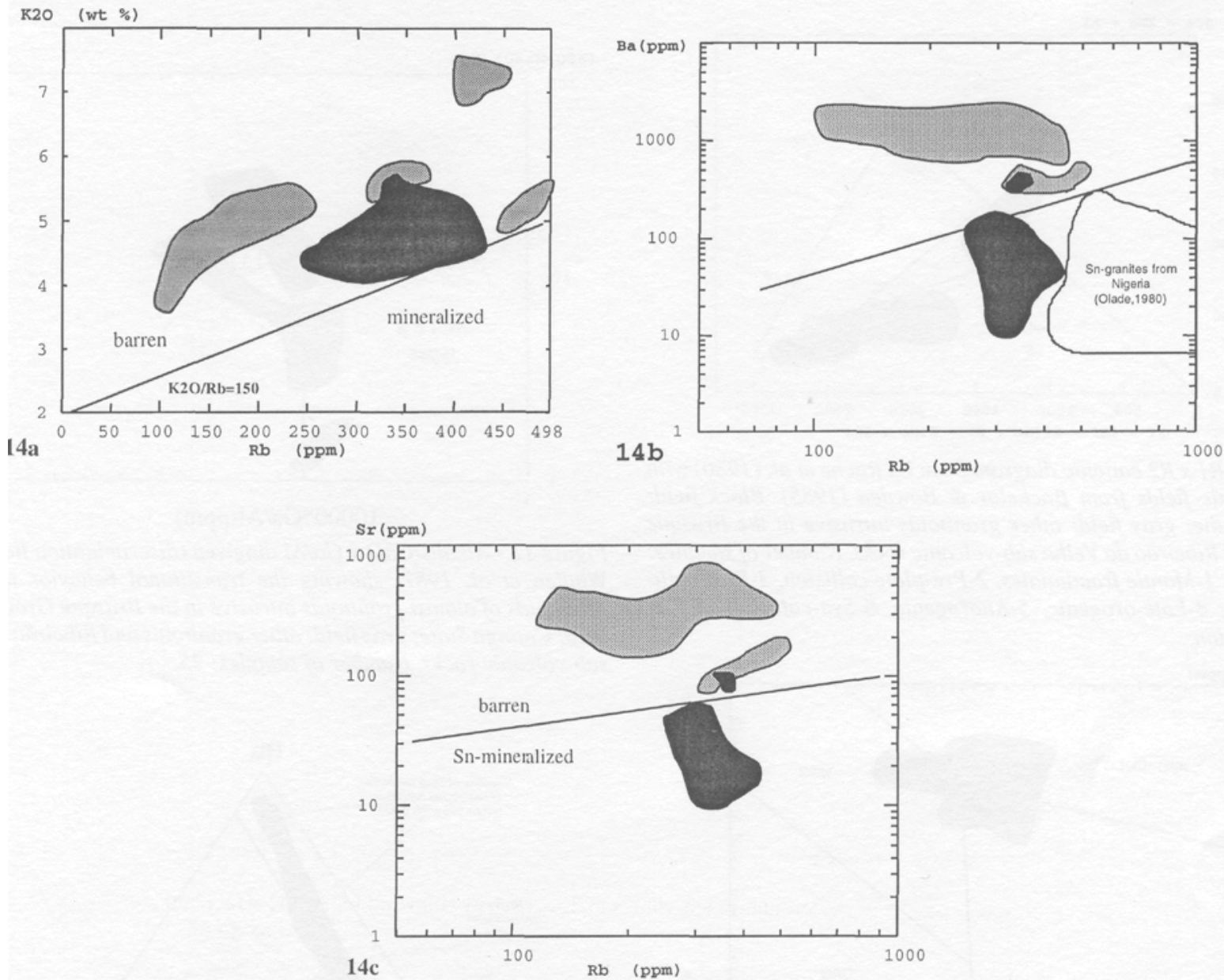

Figure 14 - Diagrams after Javier Rios et al. (1995), based on Olade's (1980) parameters, showing the Catinga Suite (black fields) potential for tin mineralization. 25 samples/diagram.

\section{References}

Basci, M.A.S. 1985. O Cinturão Dom Feliciano em Santa Catarina. Unpublished Ph.D. thesis, Universidade de São Paulo, São Paulo, 19()p.

Basci, M.A.S. 1996. O Grupo Brusque - Uma unidade em provável terreno suspeito durante o Ncoprotcro/óico, In: CONGR. BRAS. GEOL, 39, Salvador, 1996. Abstracts. Salvadora, SBG, p. 101-104.

Batchcllor, R.A. \& Bowden, P. 1985. Petrogenctic interpretation of granitic rock scries using multicalionic parameters. Chemical Geology 48:43-55.

Caldasso, A.L.S.; Cama//oto, E.; Rangrab, G.E.; Silva, M.A.S. 1988. Os granitóidcs Valsungana, Guabiruba c Faxinai no contexto dos mctamórficos do Complexo Brusque, SC. In CONGR. BRAS. GEOL.. 35, Belém, 1988. Extended Abstracts... Belém, SBG, p. 1104-1116.

Caldasso, A.L.S.; Krcbs, A.S.J.; Silva. M.A.S. 1994a. Geologia da Folha Botuvcra (SG.22-Z-D-I-2). Estado de Santa Catarina. In: CONGR. BRAS. GEOL., 38, Balneário de Camboriú, 1994. Extended Abstracts... Balneário de Camboriú, SBG, p. 78-79.

Caldasso, A.L.S.; Krcbs, A.S.J.; Silva, M.A.S. 1994b. Geologia da Folha Brusque (SG.22-Z-D-1I-1), Estado de Santa Catarina. In: CONGR. BRÁS. GEOL., 38. Balneário de Camboriú, 1994. Extended Abstracts... Balneário de Camboriú, SBG, p. ?

Castro, N.A.; Basci, M.A.S. 1995. Caracterização pcirográfica c gcoquímica das rochas granitóides inlrusivas no Grupo Brusquc-SC. In: SIMP. SUL-BRAS. GEOL, 6, Porto Alegre, 1995. Extended Abstracts... Porto Alegre, SBG, p. 82-84.

Castro, N.A. 1997. Contribuição ao conhecimento geológico-metttlogenético associado aos granitóides intrusivos no Grupo Bntsque-SC com base em informações geológicas. aerogamaespectrométricas e LANDSATfiM-5. Unpublished M.Sc. thesis, Universidade Estadual de Campinas, Campinas, $139 \mathrm{p}$.

Chappcl, B.W.; White, A.J.R. 1974. Two contrasting granite types. Pacific Geology 8:173-174.

Daoud, W.E.K. \& Anlonicto Jr.. A. 1985. Geologia do granilo cslamTcro Água Boa-Pilinga (AM). In: SIMP. GEOL. AMAZÔNIA. 2. Belém, 1985. Extended Abstracts... Bclcm, SBG, p. 17-33.

Dostal, J. \& Chattcrjcc. A. K. 1995. Origin of lopaz.-bcaring and related pcraluminous granites of the Late Devonian Davis Lake pluton, Nova Scotia, Canada: Crystal versus fluid fractionation. Chemical Geology 123:67-88.

El Boscily, A.M. \& ELSokkary, A.A. 1975. The relation between Rb, BaandSrin granitic rocks. Chemical Geology 16(3):207-219.

Harris, N.B.W.; Pcarcc, J.A.; Tindlc, A.G. 1986. Gcochcmical characteristics of collision-zone magmatism. In: COWARD, M.P.; RIES, A.C. (Eds.) Collision tectonics. Geol. Soc. Special Publ. 19:67-81.

Isslcr, R.S. 1987. Granitos c granitóides da região sul: sistema molibdenita granilo. In: First CBQq, p. 153-169.

Javier Rios, F.; Villas, R.N.N.; Dall'Agnol R. 1995. O Granilo Serra dos Carajás: I. Fácics Pctrográficas c Avaliação do Potencial Mctalogenctico para Eslanho no Sclor Norte. Rev. Bras.Geoc. 25(1):2()-31.
Lamcyrc, J. \& Bowden, P. 1982. Plutonic rock types series: Discrimination of various granitoid scries and related rocks. J Vulc. Geoth. Res. 14:169-186.

La Roche, H.; Lctcrricr, J.; Grand Claude, P.; Marchai, M. 1980. A classification of volcanic and plutonic rocks using R1-R2 diagrams and major clement analyses - ils relationships with current nomenclature. Chem. Geol. 29:183-210.

Manlovani, M.S.M.; Hawkcsworth, C.J.; Basci, M.A.S. 1987. Nd and Pb isolo'pc bearing on the crustal evolution of southeastern Bra/il. Rev. Bras. Geuc. 17:263-268.

Middlemost, E.A.K. 1985. Magmas amiMagmatic Rocks. Essex, Longman Group Limited, 193p. Miyashiro, A. 1978. Nature of alkalic volcanic rock scries. Conlr. Min. Petrol. 2:91-104.

Oladc, M.A. 1980. Gcochcmical characteristics of tin-bearing and tin-barren granites, Northern Nigeria. Earn. Geol. 75:71-82.

Pearcc, T.H.; Harris, N.B.W.; Tindlc, A.D. 1984. Trace clement discrimination diagrams for the tectonic interpretation of rocks. J. Petrol. 25(4):956-983.

Pclachin, P.; Daoud, W.E.K, 1988. Pctrografia, Petroquímica c Mincrali/açõcs dos Granitos Estanífcros de São Francisco, Aripuanã-MT. In: CONGR. BRÁS. GEOL., 35, Belém, 1988. Extended Abstracts... Belém, SBG, v. 3, p. 1174-1182.

Schul/ Jr., A.; Albuquerque, L.F.F.; Giffoni, L.E. 1969. Geologia da Quadrícula de Rio do Sul. SC. Porto Alegre: DNPM, $109 \mathrm{p}$. (Unpublished data)

Silva, M.A.S.; Krcbs, A.S.J. 1980. Projeto wolframita no Estado de Santa Catarina. Porto Alegre. DNPM/CPRM (1), 223 p. (Unpublished data)

Silva, M.A.S.: Camo///alo. E.; Krebs, A.S.J.; Silva, L.C. 1986. Depósito de wolframita de Cerro da Catinga, Nova Trento, Santa Catarina. In: DNPM 1986. Principais depósitos minerais do Brasil, v. 2, p. 417-426.

Streckciscn, A. 1976. To each plutonic rocks ils proper name. Earth Sei. Rev. 12(1): 1-33.

Sun, S.S. 1982. Chemical composition and origin of the Earth's primitive mantle. Geocli. Cosmoch. Acta 46:179-192.

Trainini, D.R.; Dias, A.A.; Krcbs, A.S.J.; Souza. E.G.; Capclcli, L; Toniolo, J.A.; Silva. L.C. da; Silva, M.A.S. 1978. Projeto Vitlal Ramos-Biguacit. Porto Alegre, DNPM/CPRM, 303p. (Unpublished data)

Whalen, J.B.; Curric, K.L.; Chappel, B.W. 1987. A-type granites: gcochemical characteristics, discrimination and petrogencsis. Contr. Min. Petr. 95:407-419.

White, A.J.R. 1992. Granite handbook: description, genesis, some associated ore deposits: In CONGR. BRAS. GEOL., 37, São Paulo, 1992. Short course. São Paulo, SBG, 109 p.

Wilson, M. 1991. Igneous Petrogenesis: A Global Tectonic Approach, London, Unwin Hyman Ltd., $466 \mathrm{p}$.

Manuscrito A-1046

Recebido em 20 de novembro de 1997 Revisão dos autores em 30 de agosto de 1998 Revisão aceita emIS de novembro de 1998 